\title{
Simulation analysis using CFD on vibration behaviors of circular cylinders subjected to free jets through narrow gaps in the vicinity of walls
}

\author{
K. Fujita \\ Osaka City University, Osaka-City, Osaka, Japan
}

\begin{abstract}
In this paper, the vibration of circular cylinders subjected to a cross-flow jetted from a narrow gap that exists in the same fluid (hereafter called jet flow or gap flow) is investigated. A problematic example of such jet flow-induced vibration is the vibration damages of fuel rods subjected to the jet flow from the gap between core baffle plates in nuclear power plants.

The numerical simulation analysis using a commercial computational fluid dynamics tool (hereafter called CFD) on the vibration of a single circular cylinder and a circular cylinders array composed of six cylinders is performed. In CFD simulation analysis, the equation of continuity, Navier-Stokes equations, are transformed in a discrete system using finite element methods. As for the boundary between structures and fluid, the arbitrary Lagrangian-Eulerian method is adopted. Taking the relative position between a cylinder and the axis of jet flow, and the gap width of jet flow as parameters, the vibration behaviors of cylinders subjected to jet flow are investigated. Furthermore, the validity of the simulation analysis is checked comparing with the experimental results that have already been reported by one of the authors.
\end{abstract}

Keywords: free jet, jet flow induced-vibration, vortex shedding vibration, fluid elastic vibration, CFD simulation, stability analysis.

\section{Introduction}

For circular cylinders subjected to uniform cross-flow, large-amplitude vibration due to vortex shedding is observed when the flow velocity reaches a critical value, above which the circular cylinders become unstable again and undergo large- 
amplitude vibrations. Many papers on these problems have been published by Connors [1], Tanaka et al. [2], Chen [3], Fujita and Wakita [4] so on.

In contrast, there have been very few studies on circular cylinders subjected to cross-flow which is not uniform, but jetted from a narrow gap, that is free jet cross-flow (hereafter called jet flow or gap flow). Fujita et al. [5], Johnson and King [6] have studied the vibration of fuel rod assemblies subjected to the gap flow through the baffle plates in nuclear reactors, and a study on pressure drop due to gap flow was performed by Eguchi and Yagawa [7], and an experimental study on the vibration of circular cylinders subjected to jet cross-flow changing many parameters have been presented by Fujita et al. [8].

In this paper following Fujita and Kawabe [9], numerical simulation results using CFD on the vibration of circular cylinders subjected to jet flow varying parameters such as the relative distance between the oscillating cylinder and the axis of jet, and the width of jet flow at gaps are reported and discussed, and moreover are compared with the experimental results reported by Fujita et al. [8]. As a trouble example of flow-induced vibration, the vibration trouble of fuel rods due to the jet flow from gaps between core baffle plates in nuclear reactors have been listed up.

\section{Numerical simulation analysis}

The numerical simulation analysis is performed using a commercial computational fluid dynamics ( hereafter called CFD) code. In CFD analysis, an equation of continuity, Navier-Stokes equations are transformed in discrete system using finite element methods. The stream upwind method is applied for a laminar flow model, and $k-\epsilon$ model for a turbulence flow model, respectively. As for the boundary between structures and fluid, the arbitrary Lagrangian-Eulerian method (hereafter called ALE method) is adopted for reducing numerical error due to the distortion of meshes for a fluid domain when structures move. A fluid is assumed to be incompressible. A flow is treated as two dimensional flow and unsteady flow.

The equation of continuity and Navier-Stokes equations are as follows:

$$
\begin{gathered}
\nabla \cdot \vec{u}=0 \\
\frac{\partial \vec{u}}{\partial t}+(\vec{u} \cdot \nabla) \vec{u}=-\frac{1}{\rho} \nabla p+v \nabla^{2} \vec{u},
\end{gathered}
$$

where $\vec{u}$ is velocity vector, $t$ is time, $\rho$ is density of fluid, $p$ is fluid pressure and $v$ is dynamic viscosity of fluid.

\section{Simulation analysis of two-dimensional free jet flow}

The simulation analysis on flow velocity distribution of free jet flow using the commercial CFD code is performed. The number of mesh is 3573 , and the region 


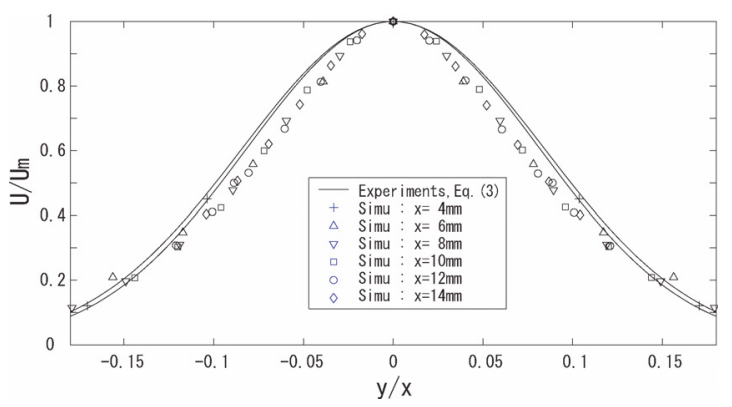

Figure 1: Velocity distribution of plane turbulent free jet.

in the vicinity of the axial direction of jet is meshed finely. The transverse velocity distributions of jet have been reported experimentally by Zijnen in Rajaratnam [10]. Figure 1 shows the comparisons between the numerical simulations and experiments done by Zijnen. In the fully developed flow region of jet, it has been experimentally found that the velocity distribution could also be represented satisfactorily by a Gaussian curve. Zijnen found that:

$$
\frac{u}{u_{m}}=\exp \left(-\bar{a} \lambda^{2}\right)
$$

where $u$ is a velocity of free jet at an axial distance $x . u_{m}$ is a maximum velocity of free jet on the axis. $\bar{a}=70.7 \sim 75.0$, and $\lambda=y / x, y$ denotes the transverse distance perpendicular to the axial direction from the axis of free jet . $x$ denotes the axial distance between the gap position of free jet and the observing position. In this figure, solid lines show the results of Eq. (3) best fitted on experiments and many symbols such as,$+ \triangle$ so on show the numerical simulations changing $x$ values to be $4 \mathrm{~mm}-14 \mathrm{~mm}$ by CFD. We find that near the axis of the jet, the CFD simulations appear to be slightly inferior, whereas in the outer region to be in a good agreement with Eq. (3).

The width $y$ of jet at an axial distance $x$ can be said to be proportional to the value of distance $x$ theoretically based on Navier-Stokes equations. Moreover, the maximum flow velocity of free jet at an axial distance $x$ can be said theoretically to be inverse proportional to the root of an axial distance $x$ from the gap position of free jet after the potential core disappears at $x=6 \mathrm{~h}$, as the momentum flux of jet must be conserved when the fluid pressure is same everywhere in the region of free jet flow. 


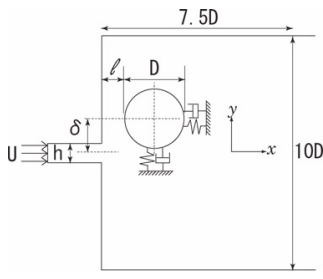

(a) Model

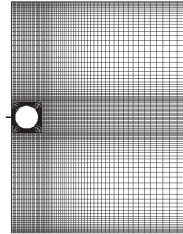

(b) Mesh for fluid

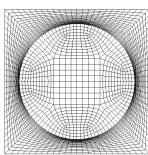

(c) Mesh for structure

Figure 2: Model and mesh for calculation.

\section{Simulation analysis of a single cylinder}

\subsection{Modeling}

Figure 2 illustrates the dimensions of a single cylinder located near rigid wall subjected to jet flow, and the mesh map of CFD analysis for both fluid region and structure region, respectively. The cylinder is supported by a spring-damper system two-dimensionally (hereafter called elastic cylinder). The diameter of cylinder is $D=16 \mathrm{~mm}$, and $l=2.4 \mathrm{~mm}$. The time step is adopted to be $0.002 \mathrm{sec}$, and the calculation time is about $2 \mathrm{sec}$. The total number of mesh is about 12484 .

\subsection{Influence of relative position $\delta$ between jet flow and cylinder}

Figure 3 shows time history waves on the vibration response of a single cylinder for various jet velocities at gaps, where the relative position of cylinder to the axis of jet vertically as shown in Fig. 2(a) is varied as $\delta=0,5.7 \mathrm{~mm}$. That is, $\delta=0 \mathrm{~mm}$ means jet cross-flow attacks a cylinder center directly, and $\delta=5.7 \mathrm{~mm}$ means a jet flow is positioned about $45 \mathrm{deg}$. to a cylinder centerline. The gap width $h$ from which a jet comes is maintained as $h=0.68 \mathrm{~mm}$.

Figure 4 shows the relationship between the displacement amplitude of cylinder and the jet velocity at gaps as for the same calculation parameters as shown in Fig. 3. In Fig. 4, the experimental values are also plotted. In case of $\delta=0 \mathrm{~mm}$ in Fig. 3(a), Fig. 4(a), the displacement amplitude is recognized to become large abruptly at the jet flow velocity of gap $U=8 \mathrm{~m} / \mathrm{s}$ in simulations and $U=9 \mathrm{~m} / \mathrm{s}$ in experiments, and the vibration loci of simulations have a good agreement with experiments.

In case of $\delta=5.7 \mathrm{~mm}$ in Fig. 3(b), Fig. 4(b), a large amplitude vibration occurs first at lower flow velocities, however, this vibration soon disappears with increasing the jet flow velocity, and at higher flow velocities $U=9 \mathrm{~m} / \mathrm{s}$, the cylinder begins to vibrate again in simulations and experiments. In case of $\delta=5.7 \mathrm{~mm}$, the average line of time history waves at higher flow velocities is shifted from zeroline, not at lower flow velocities. Observing the vibration responses in lift and drag directions although the time history waves in drag direction(x) is omitted here, this phenomenon can be said to be due to the effect based on Bernoulli's theorem for 


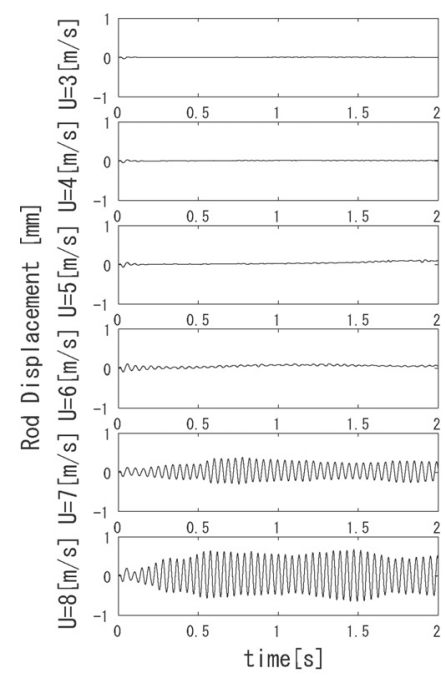

(a) $\delta=0[\mathrm{~mm}]$

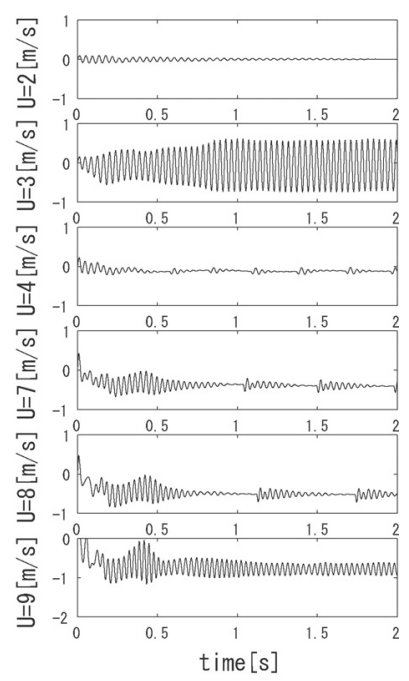

(b) $\delta=5.7[\mathrm{~mm}]$

Figure 3: Time history waves of elastic cylinder (y-direction).

pressure distribution between the rigid wall and an elastic cylinder, and the influence of drag force of jet flow. In the case of $\delta=0 \mathrm{~mm}$, these shifting phenomena appear only in the time history of drag direction at higher flow velocities region. And also, vibration loci are found to show a good agreement between simulations and experiments.

\subsection{Influence of gap width $h$ of jet flow}

Figure 5 shows the relationship in simulations between the vibration displacement of cylinder and the jet flow velocity changing the gap width as $h=5,10,20,30 \mathrm{~mm}$, where $\delta=5.7 \mathrm{~mm}$. In case of the gaps with $h=5,10,20 \mathrm{~mm}$, large amplitude vibrations, which are considered to be due to vortex shedding resonance, appears at lower flow velocities. And large unstable vibrations, which are considered to be fluid-elastic instability like galloping phenomenon, occur at higher flow velocities. However, in case of the gap width $h=30 \mathrm{~mm}$, the vibration peak at lower flow velocities can not be recognized. It is understood that the vibration peak in lower velocities region of jet flow is much sensitive to the relative position between the oscillating cylinder and the axis of jet, and the gap width.

Figure 6 shows the velocity vector over one period corresponding to point $\mathrm{E}$ in higher flow velocities of the case of $h=10 \mathrm{~mm}$ in Fig. 5. Observing this figure, the direction of jet flow shifts violently at the vicinity of cylinder surface. This shifting phenomenon is considered to be due to Coanda effect. And also, the effect based on Bernoulli's theorem can be recognized a little between the rigid wall and the elastic cylinder in Fig. 6. 

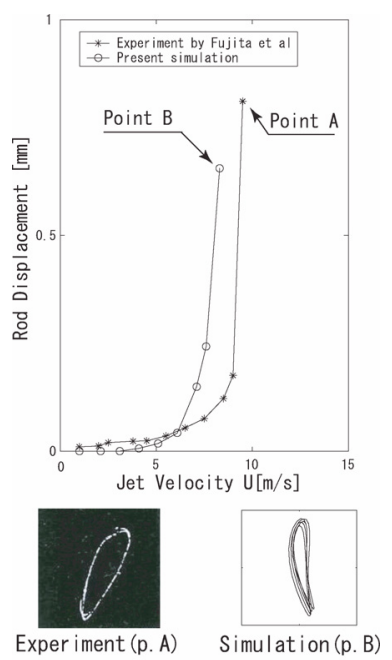

(a) $\delta=0[\mathrm{~mm}]$
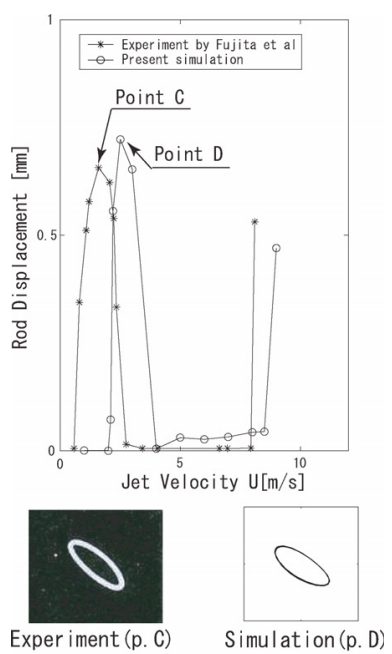

(b) $\delta=5.7[\mathrm{~mm}]$

Figure 4: Comparison between simulations and experiments.

\subsection{Consideration on vortex shedding resonance and fluid elastic vibration}

Figure 7 shows the relationship between the gap width $h$ and the reduced velocity of the vibration at the lower flow velocities region for the gap $h=0.68,1,3,5,10$, $20 \mathrm{~mm}$ in simulations in case of $\delta=5.7 \mathrm{~mm}$. The reduced velocities are estimated at the peak vibration in lower flow velocities using the predominant frequencies of a cylinder and the maximum flow velocities around a cylinder. Observing Fig. 7, the Strouhal number $S$ can be understood to be about 0.2 , and also this value is found to have a good agreement with experiments in uniform cross-flow. Moreover, the violent shifting of separation and reattachment phenomena on the surface of cylinder and the vortices shed from the separating points every period can be observed in velocity vectors and pressure contours at the peak vibration in lower velocities region, not at unstable vibration in higher velocities region. Further, the influence of stationary components of fluid forces is considered to be much small as the average lines of time history waves almost do not shift from zero-line. Judging from these results, the peak displacement appeared at rather low flow velocity region is considered to be due to a kind of vortex shedding phenomena.

On the other hand, concerning the unstable vibration at higher flow velocities, as the momentum flux in the axial direction( $x$ direction) is conserved when the fluid pressure does not change in the flowing direction theoretically, the following equation can be obtained:

$$
U^{2} h=\int_{-\infty}^{\infty} u^{2} d y=\text { const },
$$

where $U$ and $u$ are jet flow velocities at gap and distance $x$, respectively. 

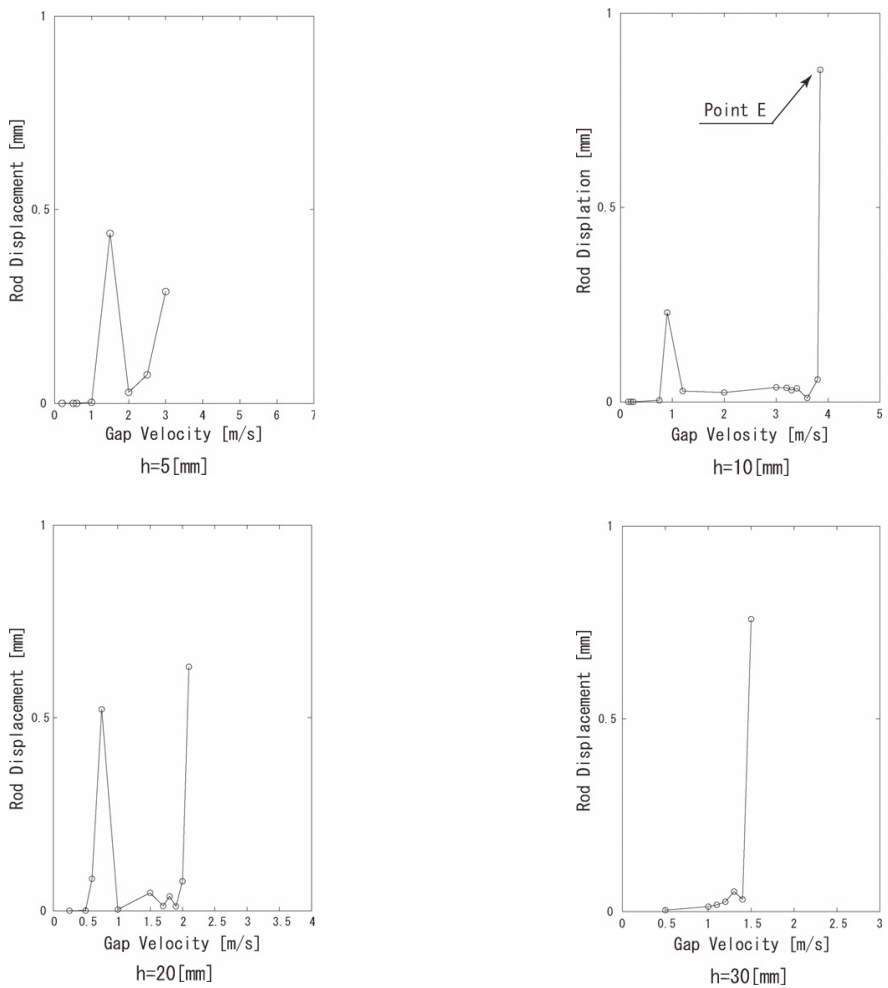

Figure 5: Vibration response (y-direction).

Therefore, let us calculate the momentum flux by using Eq. (4), at the critical flow velocity of unstable vibration in higher flow velocities region for simulation analysis. Figure 8 shows the relationship between the jet flow velocity at gap and the gap width in logarithmic coordinates. The circle symbols o show the numerical simulation results, and the solid straight lines, which incline to the right, show constant values of $U^{2} h$. Under the gap width $h=30 \mathrm{~mm}$, the circle symbols are found to be located considerably on the same line, that is the same momentum flux, so the unstable vibration at higher flow velocities region is found to be governed by a constant momentum flux of jet flow.

\section{Simulation analysis of 6 cylinders array}

\subsection{Modeling}

Figure 9 shows a modeling of 6 cylinders array for numerical simulation analysis, and a mesh map for fluid region and structure region in CFD simulation. The one cylinder subjected to jet flow directly in a cylinders array is supported with a 
92 Fluid Structure Interaction V

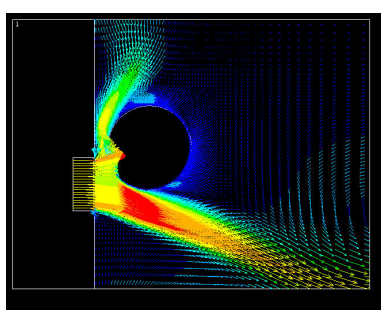

$T / 4$

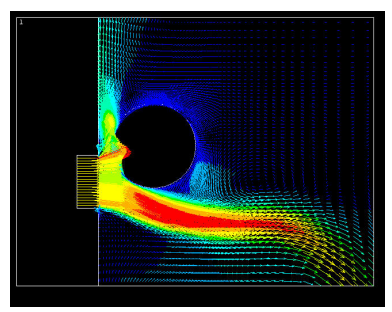

$3 T / 4$

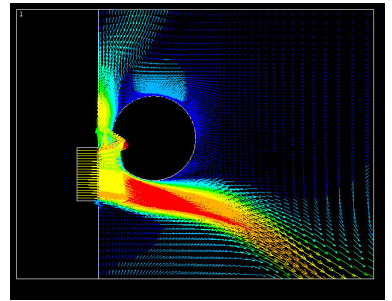

$T / 2$

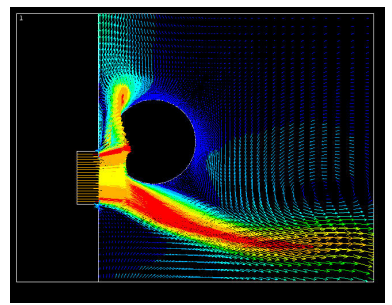

T

Figure 6: Velocity vector (Point E in Fig. 5).

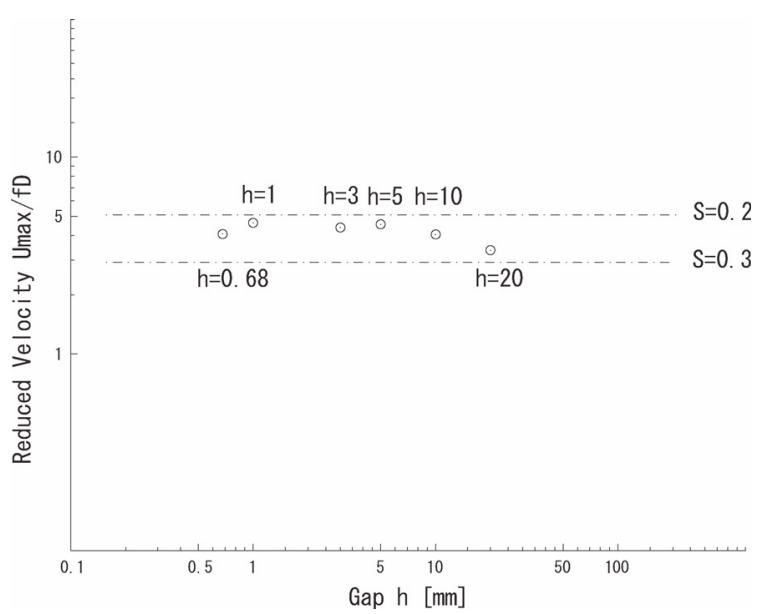

Figure 7: Strouhal number at peaks of vortex shedding vibration.

spring-damper system at the center of first raw (hereafter called elastic cylinder) as shown in Fig. 10. The others are rigid cylinders. A time step is $0.002 \mathrm{sec}$, and the calculation time is $2 \mathrm{sec}$ for each case, and the total number of mesh is 14942 . Unfortunately, there are some upper limitations as for the number of mesh in a commercial CFD code. Besides, the cylinders array in experiments compared with 


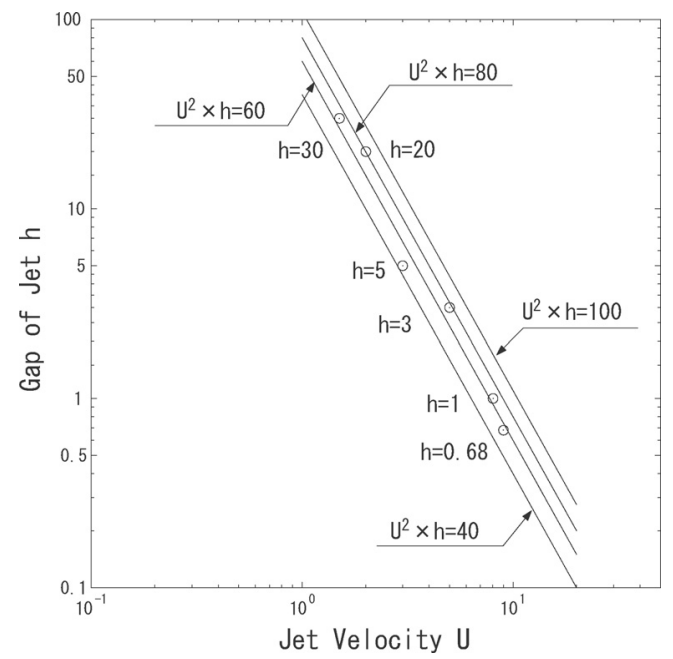

Figure 8: Relation between jet flow velocity and gap width for threshold of the fluid elastic vibration.

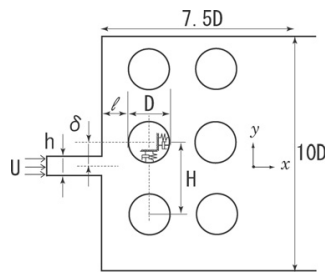

(a) Model

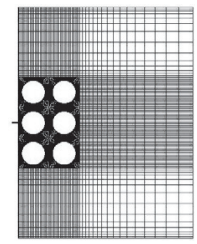

(b) Mesh for fluid

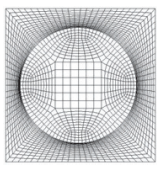

(c) Mesh for structure

Figure 9: Model and mesh for calculation.

simulations were in-line array, and all of cylinders were supported by a springdamper system.

\subsection{Influence of relative position $\delta$ and gap width $h$}

The gap width of jet flow is $h=0.68 \mathrm{~mm}$, and both $\delta=0 \mathrm{~mm}$ and $\delta=5.7 \mathrm{~mm}$ as shown in Fig. 9(a) are simulated. Figure 10 shows the relationship between the jet velocity and the displacement amplitude of the cylinder. In Fig. 10, the experimental values are also plotted for a comparison.

In case of $\delta=0 \mathrm{~mm}$, the displacement amplitude is found to become large abruptly at a jet flow velocity $U=6 \mathrm{~m} / \mathrm{s}$ in simulations and $U=7 \mathrm{~m} / \mathrm{s}$ in experiments. This unstable vibration is found to be similar with the vibration behavior of a single cylinder in higher flow velocities. 

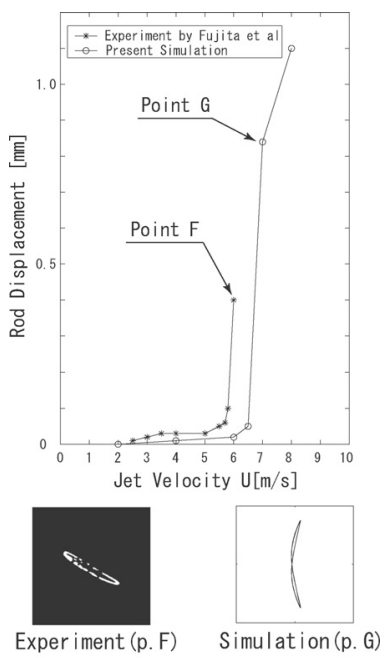

(a) $\delta=0[\mathrm{~mm}]$
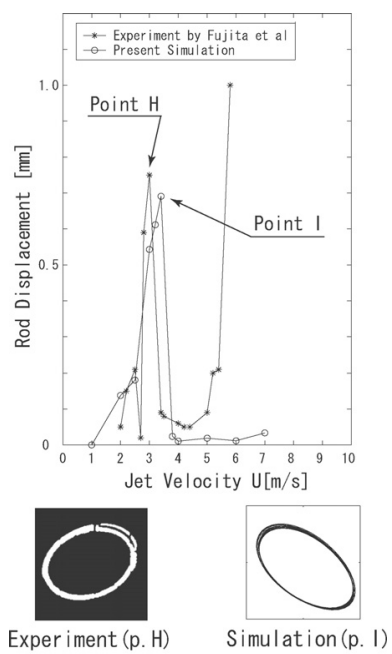

(b) $\delta=5.7[\mathrm{~mm}]$

Figure 10: Comparison between simulations and experiments.

In case of $\delta=5.7 \mathrm{~mm}$, a peak vibration of large amplitude appears at relatively low flow velocity $3.5 \mathrm{~m} / \mathrm{s}$ in simulations and $3 \mathrm{~m} / \mathrm{s}$ in experiments. And also, at the peak vibration in lower velocities region, the violent shifting of separation and reattachment phenomena on the surface of cylinder and the vortices shed from the separating points every period can be observed in velocity vectors and pressure contours in numerical simulations. However, the unstable vibration at higher flow velocities appeared in experiments could not be simulated well numerically. These phenomena also resemble to those of a single cylinder. Besides, we must note that the only one cylinder is elastic in simulation although all of cylinders was elastic in experiments.

Moreover, we calculate the Strouhal number changing the gap width in simulations, it is also found to be about 0.2 as same as that in uniform flow.

\section{Conclusions}

The following conclusions can be obtained. The vibration phenomena of circular cylinders close to rigid wall, which is subjected to cross-flow jetted from a narrow gap, is made to be clear by simulating numerically using CFD. The Strouhal number of the peak vibration at lower flow velocities is about 0.2 as same as that in uniform cross-flow. This vibration at lower flow velocities can be concluded to be due to a kind of vortex shedding phenomena. The unstable vibrations which occur abruptly in higher flow velocities of jet are considered to be fluid-elastic instability like galloping phenomenon concerning a single cylinder. It is found that a critical flow velocity at higher flow velocities region is governed by a constant momentum 
flux of jet flow. These simulation results show considerably a good agreement with the experimental results reported by one of authors.

\section{References}

[1] Connors, H. J., Fluid-elastic vibration of tube arrays excited by cross flow. In Flow-Induced Vibration in Heat Exchangers(ed. D. D. Reiff), New York: ASME, 1970.

[2] Tanaka, H., and Takahara, S., Fluid elastic vibration of tube array in cross flow. Journal of Sound and Vibration, 77, pp. 19-37, 1981.

[3] Chen, S.S., Instability mechanisms and stability criteria of a circular cylinders subjected to cross flow; part1: theory. ASME Journal of Vibration, Acoustics, Stress and Reliability in Design, 105, pp. 253-260, 1983.

[4] Fujita, K. and Wakita, T., Hybrid analysis method using CFD and analytical solutions on fluid elastic vibration of circular cylinder arrays. Fluid Structure Interaction and Moving Boundary Problems IV, WIT Press, pp.247-257, 2007.

[5] Fujita, K., Ito, T., Shiraki, K., Kondo, Y., Teranishi, T., Shiba M. and Yamasaki, Y., Flow-induced vibration of PWR fuel assembly by baffle leakage cross-flow. Transactions 6th International Conference of Structural Mechanics in Reactor Technology, B6/8, pp. 1-8, 1981.

[6] Johnson, D. K., and King, K. A., Fuel rod vibration due to cross flow through narrow gaps. Transactions 7th International Conference of Structural Mechanics in Reactor Technology, B5/9, pp. 297-304, 1983.

[7] Eguchi,Y. and Yagawa, G., Analysis of baffle jet phenomena in pressure vessel. Journal of Pressure Engineering 20-5, pp. 24-28(in Japanese), 1982.

[8] Fujita, K., Ito, T., and Kohno, N., Experimental study on the vibration of circular cylinders subjected to cross-flow jetted from a narrow gap. Journal of Fluids and Structures, 4, pp. 99-124, 1990.

[9] Fujita, K., Kawabe, K., Vibration behavior of circular cylinders subjected to jet-flow from a narrow gap. Proc. of the 8th International Conference on Flow-Induced Vibration, pp. 545-550, 2008.

[10] Rajaratnam, N., TURBULENT JETS, Elsevier scientific publishing, pp. 2122, 1976. 